\title{
EDITORIAL
}

\section{Early cancer diagnosis: reaching targets across whole populations amidst setbacks}

Early diagnosis of cancer, followed by timely and appropriate therapy, are the cornerstones of the secondary prevention of cancer, thus the NHS has set a 2028 target to achieve 75\% early stage (TNM I/II) at cancer diagnosis. In this context, Barclay et al. evaluated overall, sex, age and deprivation-group-specific progress towards this target based on 202,000 cancer patients diagnosis in 2015. Herein, we discuss their findings which form a valuable pre-COVID-19 pandemic status. We discuss the impact of the pandemic and the efforts being made in innovative early detection and diagnosis research.

British Journal of Cancer (2021) 124:1181-1182; https://doi.org/10.1038/s41416-021-01276-2

\section{MAIN}

Progress in the co-important secondary prevention elements of early diagnosis and successful treatment have paved the way for the doubling of cancer survival and improved quality of life in cancer survivors in high income countries over the past few decades. Because early diagnosis is an essential enabler of curative treatment, in its Long Term Plan the UK NHS set a target to achieve $75 \%$ early stage (TNM stage I/II) cancer by 2028 . It is within this context that Barclay, Lyratzopoulos and colleagues ${ }^{1}$ evaluated overall and socio-demographic group-specific progress towards this target among 202,000 cancer patients diagnosed with 10 common solid cancers in England in 2015. In this robust analysis, $57 \%$ of patients were diagnosed at early stages, i.e., 18 absolute percentage points $(\mathrm{pp})$ short of the target. The analysis specifically quantified the extent of inequities associated with deprivation, older age (>65 years) and sex (for most cancers, men had later stage than women), and predicted that, beyond the potential elimination of these inequities, there would still be a 14-pp shortfall from the NHS target. However, this overall picture varied immensely by cancer type: bladder, breast, endometrial and melanoma had reached the $75 \%$ early stage target in 2015 , renal and prostate cancers were a few percentage points under $60 \%$, colon and rectal $43-45 \%$ and ovary and lung under $30 \%$.

The findings identify demographic groups needing specific attention to improve early stage at diagnosis, including male and older age groups for renal cancer, more deprived groups for colon and rectal, older and deprived groups for ovary and prostate and men for lung cancer. For prostate cancer, they found that addressing inequities linked to deprivation would result in a $9.1 \%$ absolute increase in the percentage of patients diagnosed with stage $\mathrm{I} / \mathrm{II}$ cancer. ${ }^{1}$ This is substantial, but also demonstrates how, in the absence of clear evidence for a prostate cancer screening programme, that socioeconomic differences in cancer awareness and men self-referring for routine prostate-specific antigen testing, is likely to be contributing to widening inequities in prostate cancer outcomes. ${ }^{2}$ Addressing this gap will therefore not be straightforward. Across the 10 cancers, the inequities linked to older age contributed to the greatest percentage $(5.1 \%)$ of the 2015 late stage cancers, followed by inequities linked to deprivation $(2.7 \%)$, then sex $(2.0 \%)$. This older population will need further attention as the growing cancer burden predicted in the UK is largely due to the growing and ageing population. ${ }^{3}$ Furthermore, age gaps in survival already exist. ${ }^{4}$

These 2015 findings must now be put into perspective of the current major upheaval in cancer care. In a year when the severe acute respiratory syndrome Coronavirus-2 (COVID-19) pandemic has severely disrupted lives, movement, health seeking behaviours and health systems in England and beyond, the present article ${ }^{1}$ provides a comprehensive benchmark of the pre-pandemic status. In the UK, following commencement of national COVID-19 pandemic lockdown measures (16 March 2020), cancer screening was suspended, routine referral deferred or cancelled and large declines in the 2week-wait urgent referrals for diagnostic workup of suspected cancers were observed. For example, in England, between March and August 2020, there were 14,000 fewer referrals for suspected lung cancer, i.e., $60 \%$ of pre-lockdown referrals, probably because of the similarity of initial symptoms with those for mild COVID-19. ${ }^{5}$ Declines in referrals were not restricted to lung cancer, but were seen for several cancer types. Fewer diagnostic investigations were also observed, with endoscopy activity in the period up to the end of May 2020 reduced to $12 \%$ of pre-COVID levels. ${ }^{6}$ This decrease in referrals and in diagnostic investigation implies an eventual later diagnosis and possible reversal or at most stagnation of progress to achieve earlier stage at diagnosis at the population level.

Whilst it is too early to assess this pandemic's impact on cancer survival due to delays in diagnosis and treatment, modelling studies provide valuable assessments of the potential excess deaths. Using a route-to-diagnosis framework, Maringe et al. ${ }^{7}$ have estimated the impact of pandemic lockdown measures and changes in patient behaviour and availability of services for breast, colorectal, lung and oesophageal cancer. They modelled a shift from diagnoses via routine routes, including routine workup and screening, to the urgent 2-week-wait or emergency referrals operating at varying capacity levels, and found between 7.9 to $9.6 \%$ more deaths for breast, $\sim 16 \%$ for colorectal, $\sim 5 \%$ for lung and $6 \%$ for oesophageal cancer. In total, over 3300 additional COVID-19 delay-related deaths would occur for these four cancers, equating to $\sim 60,000$ years of life lost due to avoidable deaths. These results are considered conservative as they only refer to four tumour types and do not account for the impact of treatment delay or sub-optimal treatment delivery on mortality. 
During the pandemic's upheaval, the 2015 socio-demographic groups who were more vulnerable to a later cancer diagnosis need to be paid particular attention to avert yet further disadvantage. Some of these groups, notably the elderly and the most deprived, may also be disproportionately affected by further late cancer diagnosis through their greater probability of lockdowns and shielding put in place to protect against COVID-19 mortality risks. The situation is likely to be exacerbated by a failure to provide public health messaging that accurately conveys the magnitude of risks of severe illness from COVID-19 infection compared with the risks of not seeking healthcare advice if symptomatic from cancer. In addition, changes in health seeking behaviour and the increasing necessity to self-navigate a complex and dynamically changing healthcare system (e.g. increasing remote consultations) could widen gaps in individuals receiving timely access to diagnostic services and cancer treatment. For example, there has also been a change in the geographical provision of access to services due to the creation of COVID-19 free surgical pathways, ${ }^{9}$ with previous studies demonstrating a differential impact of increasing travel burden on access to cancer treatment for individuals from lower socioeconomic groups. ${ }^{10}$ Similarly, the welfare impacts of rising unemployment and income inequality from the COVID-19 pandemic may disproportionately impact on lower socioeconomic groups and has been associated with rises in cancer mortality following previous economic downturns. ${ }^{11}$ In short, the COVID-19 pandemic will have undoubtedly hindered progress towards the 2028 target of $75 \%$ of cancers diagnosed at early stages, but actions to achieve a recovery should be expected and prioritised. When possible, an updated comparative analysis of Barclay et al.'s 2015 situation to a period including and beyond the pandemic will be informative.

Notwithstanding, even had the pandemic not occurred, further improvements in early stage at cancer diagnosis are needed and Barclay et al.'s analysis ${ }^{1}$ demonstrates that these are unlikely to be achieved through addressing inequities via the current routes of diagnosis alone. To this end, the Cancer Research UK Early Detection and Diagnosis Roadmap Steering Group have highlighted the need for a paradigm shift in cancer early diagnosis. ${ }^{12,13}$ The detailed roadmap calls for a more unified multi-disciplinary and multi-sector effort to advance cancer early detection and diagnosis. It outlines a range of avenues for investigation to aid early diagnosis-from cancer biology, including precancer evolution and circulating tumour DNA, to risk prediction models, artificial intelligence, modern technological advances and finally commercialisation of products for scale-up and community reach. This roadmap holds a promising future for the early detection and diagnosis of cancer, advances that should be designed and subsequently evaluated to reach the entire population and especially demographic groups prone to late diagnosis.

\section{DISCLAIMER}

Where authors are identified as personnel of the International Agency for Research on Cancer/World Health Organization, the authors alone are responsible for the views expressed in this article and they do not necessarily represent the decisions, policy or views of these organisations.

\section{ACKNOWLEDGEMENTS}

None.

\section{AUTHOR CONTRIBUTIONS}

V.M. and A.A. jointly drafted this article.

\section{ADDITIONAL INFORMATION}

Ethical approval and consent to participate Not applicable.

Consent to publish Not applicable.

Data availability The article does not contain any data.

Competing interests The authors declare no competing interests.

Funding information No particular funding.

Publisher's note Springer Nature remains neutral with regard to jurisdictional claims in published maps and institutional affiliations.

Valerie McCormack (iD) and Ajay Aggarwal ${ }^{2}$ ${ }^{1}$ Environment and Lifestyle Epidemiology Branch, International Agency for Research on Cancer, Lyon, France and ${ }^{2}$ Department of Health Services Research and Policy, London School of Hygiene \& Tropical Medicine, London, UK Correspondence: Valerie McCormack (mccormackv@iarc.fr)

\section{REFERENCES}

1. Barclay, M. E., Abel, G. A., Greenberg, D. C., Rous, B. \& Lyratzopoulos, G. Sociodemographic variation in stage at diagnosis of breast, bladder, colon, endometrial, lung, melanoma, prostate, rectal, renal and ovarian cancer in England and its population impact. Br J Cancer https://doi.org/10.1038/s41416-021-01279-z (2021).

2. Dasgupta, P., Baade, P. D., Aitken, J. F., Ralph, N., Chambers, S. K. \& Dunn, J. Geographical variations in prostate cancer outcomes: a systematic review of international evidence. Front. Oncol. 9, 238 (2019).

3. Smittenaar, C. R., Petersen, K. A., Stewart, K. \& Moitt, N. Cancer incidence and mortality projections in the UK until 2035. Br. J. Cancer 115, 1147-1155 (2016).

4. De Angelis, R., Sant, M., Coleman, M. P., Francisci, S., Baili, P., Pierannunzio, D. et al. Cancer survival in Europe 1999-2007 by country and age: results of EUROCARE-5a population-based study. Lancet Oncol. 15, 23-34 (2014).

5. Greenwood, E. \& Swanton, C. Consequences of COVID-19 for cancer care-a CRUK perspective. Nat. Rev. Clin. Oncol. 18, 3-4 (2021).

6. Rutter, M. D., Brookes, M., Lee, T. J., Rogers, P. \& Sharp, L. Impact of the COVID19 pandemic on UK endoscopic activity and cancer detection: a National Endoscopy Database Analysis. Gut https://doi.org/10.1136/gutjnl-2020-322179 (2020).

7. Maringe, C., Spicer, J., Morris, M., Purushotham, A., Nolte, E., Sullivan, R. et al. The impact of the COVID-19 pandemic on cancer deaths due to delays in diagnosis in England, UK: a national, population-based, modelling study. Lancet Oncol. 21, 1023-1034 (2020).

8. Hanna, T. P., King, W. D., Thibodeau, S., Jalink, M., Paulin, G. A., Harvey-Jones, E. et al. Mortality due to cancer treatment delay: systematic review and metaanalysis. BMJ 371, m4087 (2020).

9. Glasbey, J. C., Nepogodiev, D., Simoes, J. F. F., Omar, O., Li, E., Venn, M. L. et al. Elective cancer surgery in COVID-19-free surgical pathways during the SARS-CoV2 pandemic: an International, Multicenter, Comparative Cohort Study. J. Clin. Oncol. https://doi.org/10.1200/JCO.20.01933 (2020). JCO2001933.

10. Tataru, D., Spencer, K., Bates, A., Wieczorek, A., Jack, R. H., Peake, M. D. et al. Variation in geographical treatment intensity affects survival of non-small cell lung cancer patients in England. Cancer Epidemiol. 57, 13-23 (2018).

11. Maruthappu, M., Watkins, J., Noor, A. M., Williams, C., Ali, R., Sullivan, R. et al. Economic downturns, universal health coverage, and cancer mortality in highincome and middle-income countries, 1990-2010: a longitudinal analysis. Lancet 388, 684-695 (2016).

12. Cancer Research UK Early Detection and Diagnosis Roadmap Steering Group. The early detection and diagnosis of cancer: a roadmap to the future. https://www. cancerresearchuk.org/sites/default/files/early_detection_diagnosis_of_cancer_ roadmap.pdf (2020).

13. Crosby, D., Lyons, N., Greenwood, E., Harrison, S., Hiom, S., Moffat, J. et al. A roadmap for the early detection and diagnosis of cancer. Lancet Oncol. 21, 1397-1399 (2020). 